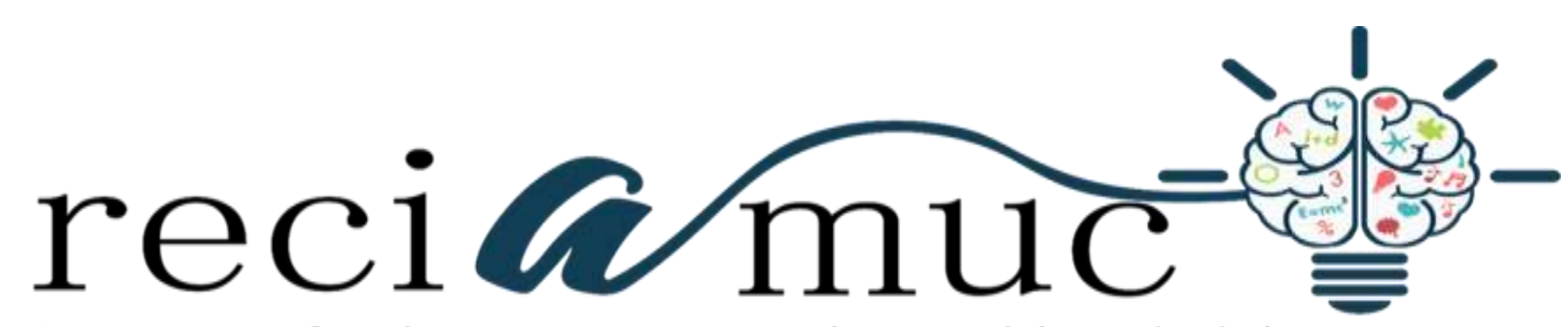

Revista cientifica de investigación actualización del mundo de las ciencias

\author{
Tania Soledad Alvarado Chávez ${ }^{\text {a; }}$ Rafael Alberto Benavides Naranjo ${ }^{\text {b; Raisa }}$ \\ Valeria Vélez Albán c; María Antonieta Touriz Bonifaz ${ }^{\mathrm{d}}$
}

Complicaciones musculoesqueléticas de las fracturas expuestas de tibia en hospital general de Babahoyo (IESS) de enero del 2015 a enero 2018

Musculoskeletal complications of tibia exposed fractures in Babahoyo General Hospital (IESS) from January 2015 to January 2018

Revista Científica de Investigación actualización del mundo de las Ciencias. Vol. 3 núm., 2, abril, ISSN: 2588-0748, 2018, pp. 84-102

DOI: $10.26820 / \mathrm{reciamuc/3.(2).abril.2019.84-102}$

\begin{abstract}
URL: http://reciamuc.com/index.php/RECIAMUC/article/view/328
\end{abstract}
Código UNESCO: 3205 Medicina Interna

Tipo de Investigación: Artículo de Revisión

(C) RECIAMUC; Editorial Saberes del Conocimiento, 2019

Recibido: 15/01/2019 Aceptado: 07/02/2019 Publicado: 01/04/2019

Correspondencia: director@reciamuc.com

a. Magister en Educación Superior; Magister en Comunicación y Desarrollo; Especialista en Traumatología y Ortopedia; Diploma Superior en Autoevaluación con Fines de Acreditación; Doctora en Medicina y Cirugía; Universidad de Guayaquil; Guayaquil, Ecuador.

b. Médico; Investigador Independiente; Guayaquil, Ecuador.

c. Médico; Investigador Independiente; Guayaquil, Ecuador.

d. Magister en Salud Publica; Magister en Epidemiologia; Medica; Universidad de Guayaquil; Universidad Católica Santiago de Guayaquil; Guayaquil, Ecuador; maria.tourizb@ug.edu.ec - maria.touriz@cu.ucsg.edu.ec 


\section{Complicaciones musculoesqueléticas de las fracturas expuestas de tibia en hospital general de Babahoyo (IESS) de enero del 2015 a enero 2018}

Vol. 3, núm. 2., (2019)

Tania Soledad Alvarado Chávez; Rafael Alberto Benavides Naranjo; Raisa Valeria Vélez Albán;

María Antonieta Touriz Bonifaz

\section{RESUMEN}

Según la Organización Mundial de la Salud, los accidentes de tránsito y sus efectos son un problema de salud pública global. Cada año más de 1,3 millones de personas mueren en el planeta por esta causa y casi la mitad de estos muertos son peatones, hombres de 15 a 44 años, cabezas de familias pobres (Organización Mundial de la Salud. Fracturas de miembros pélvicos. 2012). Los accidentes son la primera causa de mortalidad en el grupo etario de 21 a 40 años, con alto índice de amputación en traumatismo de alta energía (45\%) de la pierna, siendo los accidentes automovilísticos los que ocupan el primer lugar de frecuencia, seguidos por caídas de gran altura y accidentes violentos. Esta investigación posee un enfoque cuantitativo, no experimental de corte transversal, el método empleado es la observación directa y análisis, en donde se incluyó a 195 pacientes con diagnóstico de Fractura expuesta que cumplieron con los criterios de inclusión atendidos en el Hospital General Babahoyo de 01 de enero del 2015 a enero 01 del 2018. Resultados: Los pacientes adultos jóvenes entre 18 y 40 años, de sexo masculino fue el grupo más afectado. La mayor incidencia la posee la fractura diáfisis con $59 \%$ y Afecta sobre todo a personas jóvenes, de sexo masculino, en un accidente de tráfico, habitualmente en un contexto poli traumatológico La mayor incidencia de fractura expuesta son debido a accidentes de tránsitos con $56 \%$.

Palabras Claves: Complicaciones; Musculo Esqueléticas; Fractura Expuesta. 


\title{
Complicaciones musculoesqueléticas de las fracturas expuestas de tibia en hospital general de Babahoyo (IESS) de enero del 2015 a enero 2018
}

Vol. 3, núm. 2., (2019)

Tania Soledad Alvarado Chávez; Rafael Alberto Benavides Naranjo; Raisa Valeria Vélez Albán; María Antonieta Touriz Bonifaz

\begin{abstract}
According to the World Health Organization, traffic accidents and their effects are a global public health problem. Each year more than 1.3 million people die on the planet due to this cause and almost half of these deaths are pedestrians, men between 15 and 44 years old, heads of poor families (World Health Organization.) Pelvic limb fractures. 2012). Accidents are the first cause of mortality in the age group from 21 to 40 years, with a high rate of amputation in high-energy trauma $(45 \%)$ of the leg, with automobile accidents being the first frequency, followed by by falls of great height and violent accidents. This research has a quantitative, non-experimental cross-sectional approach; the method used is direct observation and analysis, which included 195 patients with a diagnosis of exposed fracture who met the inclusion criteria treated at the Babahoyo General Hospital of 01 from January 2015 to January 01, 2018. Results: A young adult patient between 18 and 40 years of age, male was the most affected group. The highest incidence is the diaphysis fracture with $59 \%$ and affects especially young people, male, in a traffic accident, usually in a polytraumatological context the highest incidence of fracture exposed are due to traffic accidents with $56 \%$.
\end{abstract}

Key Words: Musculoskeletal; Complications; Exposed Fracture. 


\section{Complicaciones musculoesqueléticas de las fracturas expuestas de tibia en hospital general de Babahoyo (IESS) de enero del 2015 a enero 2018}

Vol. 3, núm. 2., (2019)

Tania Soledad Alvarado Chávez; Rafael Alberto Benavides Naranjo; Raisa Valeria Vélez Albán;

María Antonieta Touriz Bonifaz

\section{Introducción.}

Las fracturas de la diáfisis de tibia se definen como la pérdida de solución de continuidad ósea en la diáfisis de tibia. Según la Sociedad Española de Traumatología y Ortopedia la fractura expuesta es toda solución de continuidad de un segmento óseo en contacto con el medio exterior, sean visibles o no los extremos fracturarías o cuando la herida está en comunicación con el foco de fractura. (SECOT, 2010)

Según la Organización Mundial de la Salud, los accidentes de tránsito y sus efectos son un problema de salud pública global. Cada año más de 1,3 millones de personas mueren en el planeta por esta causa y casi la mitad de estos muertos son peatones, hombres de 15 a 44 años, cabezas de familias pobres (Organización Mundial de la Salud. Fracturas de miembros pélvicos. 2012). Los accidentes son la primera causa de mortalidad en el grupo etario de 21 a 40 años, con alto índice de amputación en traumatismo de alta energía (45\%) de la pierna, siendo los accidentes automovilísticos los que ocupan el primer lugar de frecuencia, seguidos por caídas de gran altura y accidentes violentos (SECOT, 2010)

Dentro del estudio que se realizará en la ciudad de Babahoyo, basado en traumatismo severo de miembros inferiores, posee una alta incidencia por razones anatómicas, ya que su situación subcutánea con pobre cobertura de partes blandas y debido a la posición que ocupa la tibia dentro del esqueleto óseo tiene mayor posibilidad de recibir un traumatismo directo ya sea por accidentes de tránsito, en especial los relacionados con las motos que son actualmente la causa de lesiones con mayor incidencia en los miembros inferiores según en un estudio representan el 


\section{Complicaciones musculoesqueléticas de las fracturas expuestas de tibia en hospital general de Babahoyo (IESS) de enero del 2015 a enero 2018}

Vol. 3, núm. 2., (2019)

Tania Soledad Alvarado Chávez; Rafael Alberto Benavides Naranjo; Raisa Valeria Vélez Albán; María Antonieta Touriz Bonifaz

$59 \%$, el segundo lugar lo ocupan los accidentes laborales, en un $24.5 \%$ y el tercer lugar las caídas con un $16.3 \%$.

Las fracturas expuestas son causadas por un agente externo(1)que puede ser otro vehículo, objeto contuso, objeto cortante, asfalto, causando un efecto en la piel con gran contusión de partes blandas, bordes irregulares, trayecto anfractuoso, sucias y contaminadas (que con lleva un mayor riesgo de infección), sangrantes, (tejidos desvitalizados), impregnadas de material extraño (ropa, suciedad, plantas) o causadas por un agente interno (OMS, 2012) que son los fragmentos óseos que con su desplazamiento perforan todas las cubiertas blandas hasta la piel causando lesión de menor magnitud en las partes blandas, de bordes lisos, regulares que asemejan un corte limpio porque se producen de adentro hacia afuera (mejor pronóstico), poco sangrado y no hay presencia de cuerpos extraños, en los casos de lesiones graves de las estructuras vasculares y neurológicas, la lesión aumenta por presencia de fracturas fragmentarias y las que tienen fragmentos libres, este compromiso del periostio y la musculatura, reduce la capacidad de defensa ósea así como el potencial de consolidación, además de la presencia de microorganismos.

En el Hospital General de Babahoyo (IESS), según los reportes estadísticos internos de los últimos tres años se observa un incremento significativo del número de pacientes con fracturas expuestas en general, el presente estudio se centró específicamente en las fracturas expuestas de la tibia y analizará su asociación con los factores de riesgo o comorbilidades, al no existir información estadística precisa de su prevalencia ni identificadas las causas que motivan este incremento, se plantea una propuesta de investigación que permita en forma documentada identificar los factores de riesgo que puedan motivar un desenlace desfavorable de esta patología. 


\section{Complicaciones musculoesqueléticas de las fracturas expuestas de tibia en hospital general de Babahoyo (IESS) de enero del 2015 a enero 2018}

Vol. 3, núm. 2., (2019)

Tania Soledad Alvarado Chávez; Rafael Alberto Benavides Naranjo; Raisa Valeria Vélez Albán;

María Antonieta Touriz Bonifaz

Según el grado de exposición para el manejo terapéutico, Gustilo fue el que más estudió las fracturas expuestas y presento un estudio, tipo serie de casos de más 1825 pacientes en los que fueron tratados según su clasificación: las clasificadas en 1er y 2do grado tuvieron un mejor pronóstico que las $3 \mathrm{~b}$ y $3 \mathrm{c}$.

Es por esto la importancia en el manejo inicial de este tipo de fracturas, el cual puede mejorar su pronóstico. (Cowie \& Court-Brown, 2012)

Este estudio se realizó en pacientes que fueron al Hospital General de Babahoyo en enero del 2015 a enero del 2018, que afecta en la mayoría de los casos desde dejar de realizar actividades de la vida cotidiana, como ir a trabajar, atender a su familia, etc. y/o presentar secuelas como discapacidades físicas. Al determinar las complicaciones podremos establecer los efectos en la vida de estos pacientes para su prevención como una contribución a la sociedad.

La fractura de tibia es un reto para el cirujano ortopédico, el médico se esfuerza por salvar la vida del paciente y de la extremidad. Se centra en unir la fractura y prevenir la infección, el mantenimiento de una extremidad funcional es la meta; mientras que el riesgo implícito en las lesiones severas es la amputación (Gougoulias, Khanna, \& Maffulin, 2009)

Este estudio es conveniente porque proporciona información estadística actualizada del comportamiento demográfico, factores de riesgo y complicaciones de las fracturas expuestas de tibia en la población de pacientes del hospital general de Babahoyo (IESS). Es importante, identificar el grupo de pacientes susceptibles a complicaciones con el propósito de realizar un 


\section{Complicaciones musculoesqueléticas de las fracturas expuestas de tibia en hospital general de Babahoyo (IESS) de enero del 2015 a enero 2018}

Vol. 3, núm. 2., (2019)

Tania Soledad Alvarado Chávez; Rafael Alberto Benavides Naranjo; Raisa Valeria Vélez Albán; María Antonieta Touriz Bonifaz

manejo oportuno y adecuado en la toma de decisiones para reducir la incidencia de complicaciones o secuelas.

La relevancia social de esta investigación está dada por el análisis retrospectivo de la prevalencia de esta enfermedad en el hospital y su relación con los factores de riesgo o comorbilidades asociadas, esto permitirá identificar grupos vulnerables, implementar estrategias de prevención y transferir información actualizada sobre la enfermedad. A través de una investigación descriptiva y observacional, que identifique los factores de riesgo y determine su asociación con las complicaciones presentadas, será posible disminuir la prevalencia de complicaciones de la enfermedad y secuelas.

En el Hospital General de Babahoyo (IESS) hay una incidencia importante de personas con traumatismo de los miembros inferiores, especialmente de la pierna, zona anatómica donde está la tibia y siendo este un hueso vulnerable a traumatismo es mi interés reportar esta serie de pacientes, comentar sobre su evolución clínica, correlacionando los factores de riesgo con las complicaciones y los tratamientos implementados con los resultados clínicos.

Los tipos de fracturas expuestas según la gravedad pueden dejar secuelas crónicas que producen un desgaste emocional, económico en los pacientes como, por ejemplo, la osteomielitis, infecciones de herida quirúrgica, la cual es la más frecuente. Este desgaste económico, físico y psicológico de someterse a constantes controles podría disminuirse si realizamos una buena educación vial, (respetar las señales de tránsito, manejar adecuadamente, controles y protección), mejorar las estructuras de las avenidas, etc. 


\section{Complicaciones musculoesqueléticas de las fracturas expuestas de tibia en hospital general de Babahoyo (IESS) de enero del 2015 a enero 2018}

Vol. 3, núm. 2., (2019)

Tania Soledad Alvarado Chávez; Rafael Alberto Benavides Naranjo; Raisa Valeria Vélez Albán;

María Antonieta Touriz Bonifaz

La prevención de las complicaciones esta investigación es posible realizarla en el servicio de traumatología y ortopedia del hospital general de Babahoyo ya que se cuenta con los recursos, financieros, humanos y materiales necesario

\section{Clasificación de Gustilo y Anderson}

Sobre la base de un estudio retrospectivo y prospectivo de 1.025 fracturas abiertas, Gustilo y Anderson

\section{Tipo I de Gustilo}

Las fracturas de este tipo presentan una herida limpia de menos de $1 \mathrm{~cm}$ de tamaño con o sin muy poca contaminación. La herida se produce por una perforación de la piel de adentro afuera provocada por uno de los fragmentos de la fractura. Las fracturas tipo 1 son fracturas simples, espiroideas u oblicuas cortas.

\section{Tipo II de Gustilo}

Las lesiones presentan laceración de la piel mayor del $1 \mathrm{~cm}$. pero los tejidos circundantes tienen poco o ningún signo de contusión. No existe necrosis muscular y la inestabilidad de la fractura es de moderada a grave. 


\section{Complicaciones musculoesqueléticas de las fracturas expuestas de tibia en hospital general de Babahoyo (IESS) de enero del 2015 a enero 2018}

Vol. 3, núm. 2., (2019)

Tania Soledad Alvarado Chávez; Rafael Alberto Benavides Naranjo; Raisa Valeria Vélez Albán; María Antonieta Touriz Bonifaz

\section{Tipo III de Gustilo}

Estas fracturas presentan un daño extenso de los tejidos blandos, frecuentemente con compromiso vascular, con o sin contaminación grave de la herida y con marcada inestabilidad de la fractura debida a conminación o pérdidas óseas segmentarías.

Debido a varias características diferentes de las lesiones que se presentan en este grupo, Gustilo decidió dividirlo en tres subtipos IIIA, III B y IIIC.

\section{Tipo III A de Gustilo}

Habitualmente se producen por traumatismos de alta energía, pero todavía y a pesar de una extensa laceración o colgajos existe una suficiente cobertura cutánea del hueso fracturado.

\section{Tipo III B de Gustilo}

En contraste con el tipo III A, presenta una extensa pérdida de los tejidos blandos con desprendimiento perióstico y hueso expuesto. Estas lesiones se asocian habitualmente a contaminación masiva.

\section{Tipo III C de Gustilo}

Correspondiente a cualquier fractura abierta con lesión arterial que requiera reparación, independientemente del tipo de fractura. 


\section{Complicaciones musculoesqueléticas de las fracturas expuestas de tibia en hospital general de Babahoyo (IESS) de enero del 2015 a enero 2018}

Vol. 3, núm. 2., (2019)

Tania Soledad Alvarado Chávez; Rafael Alberto Benavides Naranjo; Raisa Valeria Vélez Albán;

María Antonieta Touriz Bonifaz

\section{Clasificación según la afectación de partes blandas}

A veces se olvida que cualquier fractura, las partes blandas adyacentes sufre los efectos del mismo traumatismo y que esto supondrá: - Un mayor riesgo de infección - Reducción del potencial de consolidación ósea - Modificación de las posibilidades terapéuticas La consideración de las lesiones de las partes blandas junto a la fractura nos servirán para establecer un pronóstico y planificar el tratamiento, y en función de estas lesiones podemos clasificar a las fracturas en abiertas y cerradas; según exista ó no comunicación de la fractura con el exterior.

\section{Clasificación de Tscherne de las lesiones de los tejidos blandos}

En la clasificación de Tscherne, las lesiones de los tejidos blandos se agrupan según la gravedad en cuatro categorías diferentes,

\section{Fractura abierta grado I (Fr. O I)}

Las fracturas de este grupo son las que tienen una laceración herida de la piel producida por un fragmento óseo desde dentro. No existe contusión de la piel o, si la hay, es mínima. Estas fracturas se producen por un traumatismo indirecto (fracturas tipo A según la clasificación de la AO). Sin embargo, en casos con herida mínima de la piel o incluso sin lesión visible de los tejidos blandos, pero con la fractura producida por un traumatismo directo, como en las fracturas tipos B y C de la clasificación de la AO, deben clasificarse como abiertas grado 11. 


\section{Complicaciones musculoesqueléticas de las fracturas expuestas de tibia en hospital general de Babahoyo (IESS) de enero del 2015 a enero 2018}

Vol. 3, núm. 2., (2019)

Tania Soledad Alvarado Chávez; Rafael Alberto Benavides Naranjo; Raisa Valeria Vélez Albán; María Antonieta Touriz Bonifaz

\section{Fractura abierta grado II (Fr. O II)}

Se caracterizan por una laceración de la piel con contusión circunferencial de la misma o de otros tejidos blandos con moderada contaminación y una lesión ósea de cualquier tipo. En este grado de la clasificación se incluye cualquier lesión grave de los tejidos blandos sin lesión de un vaso o de un nervio periférico importante.

\section{Fractura abierta grado III (Fr. O III)}

Para clasificar una fractura en grado III debe presentar un daño extenso de los tejidos blandos, a menudo con una lesión adicional de un vaso o nervio importante. Cualquier fractura que se acompañe de isquemia y conminación importante pertenece a este grupo. Por lo tanto, accidentes en granjas, lesiones por arma de fuego de alta velocidad, y síndromes compartiméntales manifiestos se clasifican como grado III por su extremado y alto riesgo de infección.

\section{Fractura abierta grado IV (Fr. O IV)}

Las fracturas abiertas grado IV corresponden a las que presentan amputaciones subtotales y totales. Las amputaciones subtotales se han definido por el «Comité de Reimplantación de la Sociedad Internacional ele Cirugía Reconstructiva» como la pérdida de continuidad de todas las estructuras anatómicas importantes, especialmente de los grandes vasos con isquemia completa. El <<puente» restante de tejidos blandos no debe exceder de $1 / 4$ de la circunferencia de la extremidad. Cualquier caso en que haya revascularización puede sólo clasificarse como fractura abierta grado llI. 


\section{Complicaciones musculoesqueléticas de las fracturas expuestas de tibia en}

hospital general de Babahoyo (IESS) de enero del 2015 a enero 2018

Vol. 3, núm. 2., (2019)

Tania Soledad Alvarado Chávez; Rafael Alberto Benavides Naranjo; Raisa Valeria Vélez Albán;

María Antonieta Touriz Bonifaz

\section{Sistema de clasificación de las lesiones de los tejidos blandos de la AO}

Descripción de lesiones de la piel (Petrisor, Bhandari, Schemitsch, \& E.)en fracturas abiertas. Lesión de la piel I0 (lntegument Open) (fracturas abiertas)

I0 1 Rotura de la piel de dentro afuera

I0 2 Rotura de la piel desde fuera $<5 \mathrm{~cm}$, bordes contusos

I0 3 Rotura de la piel desde fuera $>5 \mathrm{~cm}$, mayor contusión, bordes desvitalizados

I0 4 Contusión considerable de todo el grosor de la piel, abrasión, despegamiento extenso abierto, pérdida de piel

Descripción de las lesiones musculares y tendinosas

Lesión músculo/tendón (MT) MT 1 Sin lesión muscular

MT 2 Lesión muscular circunscrita, sólo un compartimiento MT 3 Lesión muscular considerable, dos compartimientos

MT 4 Defecto muscular, laceración tendinosa, contusión muscular extensa

¡MT 5 Síndrome compartimenta! o síndrome de aplastamiento con amplias zonas de lesión

Descripción de las lesiones neurovasculares.

Lesión neurovascular (NV) NV 1 Sin lesión neurovascular NV 2 Lesión nerviosa aislada NV 3 Lesión vascular localizada 


\section{Complicaciones musculoesqueléticas de las fracturas expuestas de tibia en hospital general de Babahoyo (IESS) de enero del 2015 a enero 2018}

Vol. 3, núm. 2., (2019)

Tania Soledad Alvarado Chávez; Rafael Alberto Benavides Naranjo; Raisa Valeria Vélez Albán; María Antonieta Touriz Bonifaz

NV 4 Lesión vascular segmentaria extensa

NV 5 Lesión neurovascular combinada, incluyendo amputación total o subtotal (Thomas P.

Ruedi, 2003)

\section{Métodos.}

Esta investigación posee un enfoque cuantitativo, no experimental de corte transversal, el método empleado es la observación directa y análisis. Es no probabilística cuantitativa, incluyó a 195 pacientes con diagnóstico de Fractura expuesta que cumplieron con los criterios de inclusión atendidos en el Hospital General Babahoyo de 01 de enero del 2015 a enero 01 del 2018

Los instrumentos por manipular fueron las historias clínicas de los pacientes. Las fichas clínicas fueron instrumentadas por los médicos residentes en la fecha que se atendió al paciente y cubren las edades, tipo de fractura, sexo, etc.

El análisis de los datos recogidos se realizó en Microsoft Excel 2010 y en el programainformático SPSS 24.0, se manipulóestadística descriptiva y no paramétrica para el análisis de datos, los cuales se expresarán en frecuencias, porcentajes.

Se examinaron los principios éticos, por lo que se clasificó como una investigación sin riesgo, ya que no existió contacto directo con los pacientes, ya que se empleó la observación indirecta a través del análisis de las historias clínicas, de la base de datos del Hospital General Babahoyo. 


\section{Complicaciones musculoesqueléticas de las fracturas expuestas de tibia en hospital general de Babahoyo (IESS) de enero del 2015 a enero 2018}

Vol. 3, núm. 2., (2019)

Tania Soledad Alvarado Chávez; Rafael Alberto Benavides Naranjo; Raisa Valeria Vélez Albán;

María Antonieta Touriz Bonifaz

\section{Resultados.}

Tabla 1. Rango de edad

\begin{tabular}{|c|c|c|}
\hline Edades & Número & Porcentaje \\
\hline $18-40$ años & 115 & $59 \%$ \\
\hline $41-60$ años & 49 & $25 \%$ \\
\hline$>60$ años & 31 & $16 \%$ \\
\hline Total & $\underline{\mathbf{1 9 5}}$ & $\underline{\mathbf{1 0 0 \%}}$ \\
\hline
\end{tabular}

Fuente: Historia clínica

Elaborado por: Los autores

Se puede observar que la edad con mayor incidencia con fractura expuesta es entre 18 y 40 con un $59 \%$.

Tabla 2. Sexo

\begin{tabular}{|c|c|c|c|}
\hline Síndrome & Frecuencia & Porcentaje Válido & Porcentaje acumulado \\
\hline Masculino & 158 & 81,0 & 81,0 \\
\hline Femenino & 37 & 19,0 & 100,0 \\
\hline Total & 195 & 100,0 & \\
\hline
\end{tabular}

Fuente: Historia clínica

Elaborado por: Los autores

El sexo que mayor incidencia posee en fractura expuesta es el género masculino con $81 \%$ mientras que el femenino con 19\%. Esto se debe a problemas terapéuticos que afectan a la tibia.

Tabla 3. Fractura 


\section{Complicaciones musculoesqueléticas de las fracturas expuestas de tibia en hospital general de Babahoyo (IESS) de enero del 2015 a enero 2018}

Vol. 3, núm. 2., (2019)

Tania Soledad Alvarado Chávez; Rafael Alberto Benavides Naranjo; Raisa Valeria Vélez Albán; María Antonieta Touriz Bonifaz

\begin{tabular}{|c|c|c|c|}
\hline Síndrome & Frecuencia & $\begin{array}{c}\text { Porcentaje } \\
\text { válido }\end{array}$ & Porcentaje acumulado \\
\hline Fractura Diáfisis & 115 & 59,0 & 59,0 \\
\hline Fractura Epífisis Inferior & 31 & 15,9 & 74,9 \\
\hline Fractura Epífisis Superior & 49 & 25,1 & 100,0 \\
\hline Total & 195 & 100,0 & \\
\hline
\end{tabular}

Fuente: Historia clínica

Elaborado por: Los autores

Se puede observar que la mayor incidencia la posee la fractura diáfisis con $59 \%$ y Afecta sobre todo a personas jóvenes, de sexo masculino, y en ocasiones en un accidente de tráfico, habitualmente en un contexto poli traumatológico.

Tabla 4. Etiología

\begin{tabular}{|c|c|c|c|}
\hline Síndrome & Frecuencia & $\begin{array}{c}\text { Porcentaje } \\
\text { válido }\end{array}$ & $\begin{array}{c}\text { Porcentaje } \\
\text { acumulado }\end{array}$ \\
\hline Accidente de Tránsito & 110 & 56,4 & 56,4 \\
\hline Caídas & 38 & 19,5 & 75,9 \\
\hline Accidentes Laborales & 47 & 24,1 & 100,0 \\
\hline Total & 195 & 100,0 & \\
\hline
\end{tabular}

Fuente: Historia clínica

Elaborado por: Los autores 


\section{Complicaciones musculoesqueléticas de las fracturas expuestas de tibia en hospital general de Babahoyo (IESS) de enero del 2015 a enero 2018}

Vol. 3, núm. 2., (2019)

Tania Soledad Alvarado Chávez; Rafael Alberto Benavides Naranjo; Raisa Valeria Vélez Albán;

María Antonieta Touriz Bonifaz

Se la etiología la mayor incidencia de fractura expuesta se debe a accidentes de tránsitos con $56 \%$, mientras que el $24 \%$ se debe a accidentes laborales, por último, con el $19 \%$ se deben a caídas.

Tabla 5. Tipo de complicaciones

\begin{tabular}{|c|c|c|c|}
\hline Síndrome & Frecuencia & $\begin{array}{c}\text { Porcentaje } \\
\text { válido }\end{array}$ & $\begin{array}{c}\text { Porcentaje } \\
\text { acumulado }\end{array}$ \\
\hline Compartimenta & 61 & 31,3 & 31,3 \\
\hline Pseudoartrosis & 8 & 4,1 & 35,4 \\
\hline Dolor Anterior de Rodilla & 25 & 12,8 & 48,2 \\
\hline Rotura de Implante & 15 & 7,7 & 55,9 \\
\hline Infección & 86 & 44,1 & 100,0 \\
\hline Total & 195 & 100,0 & \\
\hline
\end{tabular}

Fuente: Historia clínica

Elaborado por: Los autores

El tipo de complicaciones con mayor prevalencia se debe a infecciones con un 44\%, debido a que la fractura expuesta en la que los extremos óseos han penetrado la piel, hallándose el hueso en contacto con el medio exterior.

Tabla 6 accidentes de tránsito

\begin{tabular}{|c|c|}
\hline Accidentes de tránsito en los ríos & Frecuencia \\
\hline Fallecidos 2015 & 163 \\
\hline Fallecidos 2016 & 135 \\
\hline Fallecidos 2017 & 180 \\
\hline Fallecidos 2018 Marzo & 42 \\
\hline
\end{tabular}




\section{Complicaciones musculoesqueléticas de las fracturas expuestas de tibia en hospital general de Babahoyo (IESS) de enero del 2015 a enero 2018}

Vol. 3, núm. 2., (2019)

Tania Soledad Alvarado Chávez; Rafael Alberto Benavides Naranjo; Raisa Valeria Vélez Albán; María Antonieta Touriz Bonifaz

\section{Fuente: ANT}

Elaborado por: Los autores

Según los datos obtenidos de la agencia nacional de tránsito de la provincia de los ríos en el año 2017 con 180 casos de accidente de tránsito hubo, seguido por el 2015 con 163 caso reportados.

\section{Tabla 7. Lesiones por accidentes}

\begin{tabular}{|c|c|}
\hline 2015 & 1081 \\
\hline 2016 & 904 \\
\hline 2017 & 949 \\
\hline mar-18 & 164 \\
\hline
\end{tabular}

Fuente: ANT

Elaborado por: Los autores

En lo que va del año la mayor incidencia en lesiones por accidentes de tránsito fue en el 2015 con 1081 casos reportados por la agencia nacional de transito

\section{Conclusiones.}

Los resultados de esta investigación se reportaron que el sexo masculino (81\%) fue el de mayor incidencia, de los cuales el grupo etario de 18-40 años. En el estudio de Sous, el resultado fue muy parecido a los resultados obtenidos en esta investigación donde el sexo masculino prevaleció con un (89\%) entre los rangos de edad de 20-40 años con mayor prevalencia a 


\section{Complicaciones musculoesqueléticas de las fracturas expuestas de tibia en hospital general de Babahoyo (IESS) de enero del 2015 a enero 2018}

Vol. 3, núm. 2., (2019)

Tania Soledad Alvarado Chávez; Rafael Alberto Benavides Naranjo; Raisa Valeria Vélez Albán;

María Antonieta Touriz Bonifaz

fracturas expuestas mientras que otros autores, reportaron al sexo masculino 91\% (237), como el más frecuente, observando mayor cantidad en el grupo de adultos medios (40-60 años).

Los pacientes adultos jóvenes entre 18 y 40 años, de sexo masculino fue el grupo más afectado.

La mayor incidencia la posee la fractura diáfisis con $59 \%$ y Afecta sobre todo a personas jóvenes, de sexo masculino, en un accidente de tráfico, habitualmente en un contexto poli traumatológico.

La mayor incidencia de fractura expuesta es debido a accidentes de tránsitos con 56\%. con un $44 \%$ de prevalencia se encuentra las infecciones, debido a que la fractura expuesta ha penetrado la piel, teniendo el hueso contacto con el exterior.

En el 2017 se reportaron alrededor de con 180 casos de accidente de tránsito hubo, seguido por el 2015 con 163 caso reportados

Debido a que el tipo y diseño de este estudio es únicamente descriptivo, no permite establecer ninguna relación. No obstante, se ha demostrado que algunas de las variables de este estudio se hallan relacionadas.

Se recomienda realizar campañas preventivas de accidentes de tránsitos con el propósito de reducir la tasa de mortalidad como también las lesiones de fractura expuesta.

La obtención de huesos desde un banco ya que este sería un avance en la práctica clínica. 


\section{Complicaciones musculoesqueléticas de las fracturas expuestas de tibia en hospital general de Babahoyo (IESS) de enero del 2015 a enero 2018}

Vol. 3, núm. 2., (2019)

Tania Soledad Alvarado Chávez; Rafael Alberto Benavides Naranjo; Raisa Valeria Vélez Albán; María Antonieta Touriz Bonifaz

\section{Bibliografía.}

Cowie, J., \& Court-Brown, C. (2012). Focus on tibial fractures. The Journal of Bone and Joint Surgery, 1-21.

Gougoulias, N., Khanna, A., \& Maffulin, N. (2009). Open tibial fractures. Are children small adults? Hippokratia., 1. Gougoulias NE1, Khanna A, Maffulin N. Open tibial fractures. Are children small adults? Hippokratia. 2009 Jul;13(3):147-53.

OMS. (2012). Fracturas de miembros pélvicos. Organización Mundial de la Salud. [Online].

Petrisor, B., Bhandari, M., Schemitsch, \& E. (s.f.). Tibia and fibula fractures. Rockwood : 3rd, editors. Rockwood .

SECOT, S. E. (2010). Técnicas quirúrgicas en Ortopedia y Traumatología (Vol. 4). Barcelona, España: ElsevierMasson SAS.

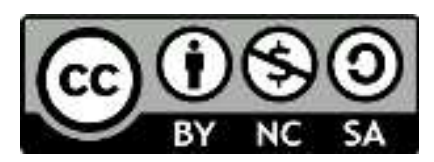

RECONOCIMIENTO-NOCOMERCIAL-COMPARTIRIGUAL

CC BY-NC-SA

ESTA LICENCIA PERMITE A OTROS ENTREMEZCLAR, AJUSTAR Y CONSTRUIR A PARTIR DE SU OBRA CON FINES NO COMERCIALES, SIEMPRE Y CUANDO LE RECONOZCAN LA AUTORÍA Y SUS NUEVAS CREACIONES ESTÉN BAJO UNA LICENCIA CON LOS MISMOS TÉRMINOS. 